\title{
Covert shifts of attention precede involuntary eye movements
}

\author{
MATTHEW S. PETERSON, ARTHUR F. KRAMER, and DAVID E. IRWIN \\ Beckman Institute, University of Illinois at Urbana-Champaign, Urbana, Illinois
}

\begin{abstract}
There is considerable evidence that covert visual attention precedes voluntary eye movements to an intended location. What happens to covert attention when an involuntary saccadic eye movement is made? In agreement with other researchers, we found that attention and voluntary eye movements are tightly coupled in such a way that attention always shifts to the intended location before the eyes begin to move. However, we found that when an involuntary eye movement is made, attention first precedes the eyes to the unintended location and then switches to the intended location, with the eyes following this pattern a short time later. These results support the notion that attention and saccade programming are tightly coupled.
\end{abstract}

Although we are free to move our attention covertly around the visual field when our eyes are at rest, the act of programming an eye movement appears to lead to an obligatory shift of covert attention to the saccade target before the eyes have even begun to move (Deubel \& Schneider, 1996; Henderson, 1992; Henderson \& Hollingworth, 1999; Henderson, Pollatsek, \& Rayner, 1989; Hoffman \& Subramaniam, 1995; Irwin \& Gordon, 1998; Kowler, Anderson, Dosher, \& Blaser, 1995; Rayner, McConkie, \& Ehrlich, 1978). For example, response targets that appear briefly between a signal to move the eyes and the initiation of the saccade are more likely to be correctly identified when they occur at the location of the saccade target than when they occur elsewhere (Deubel $\&$ Schneider, 1996). Even when instructed to attend to a specific location, response targets are more likely to be correctly reported when they coincide with the target of the eventual saccade than when they coincide with the "attended" location. This suggests that the programming of an eye movement leads to an obligatory shift of covert attention to the saccade target before the eyes have begun to move.

Previous studies suggesting this obligatory coupling have involved voluntary eye movements. For involuntary shifts of gaze, the connection between covert and overt attention is less clear. Experiments in which the eyes must remain still and attention is moved covertly have suggested that exogenous cues, such as peripheral flashes (onsets), capture attention $100 \%$ of the time (Yantis \& Jonides, 1984). ${ }^{1}$ In contrast, when eye movements have

\footnotetext{
This research was supported by Grant AG14966 from the National Institute on Aging. We thank Shawn Bolin for his assistance in running participants. We also thank Jim Juola, Martin Eimer, Tom Carr, and an anonymous reviewer for helpful comments on a previous version of this manuscript. Correspondence concerning this article should be addressed to M. Peterson, Department of Psychology, George Mason University, MS 3F5, 4400 University Dr., Fairfax, VA 22030 (e-mail: mpeters2@gmu.edu).
}

been required, onsets have led to involuntary saccades on only a minority of trials (Irwin, Colcombe, Kramer, \& Hahn, 2000; Kramer, Cassavaugh, Irwin, Peterson, \& Hahn, 2001; Theeuwes, Kramer, Hahn, \& Irwin, 1998), and the degree of capture has been shown to be modulated by top-down expectancies (Peterson, Kramer, Irwin, \& Hahn, 2002). These conflicting results suggest that either involuntary saccades and covert shifts of attention are not coupled or that the studies measuring eye movements, which can detect the presence or absence of capture on a trial-by-trial basis, were more sensitive than were the experiments on covert attention that averaged across manual response times (RTs). Mokler and Fischer (1999) have argued that attention movements do not precede involuntary saccades. They based this conclusion on the fact that participants are frequently unaware that they have made an involuntary saccade.

The following series of experiments was designed to examine the question of whether covert and overt attention are coupled when one is faced with simultaneous exogenous and endogenous cues. We used a modified version of the task used by Theeuwes et al. (1998), in which participants must move their eyes to a uniquely colored circle (the endogenous cue) and identify a small target letter that occurs within it (see Figure 1). The participants began each trial by fixating a central point that was surrounded by six white circles that contained figure- 8 premasks. After some period of time, all of the circles but one turned red and the figure- 8 premasks turned into letters. The participant's task was to move his or her eyes to the remaining white circle and to identify the small letter it contained. An additional circle, the sudden onset, was also presented at a previously unoccupied location when the original circles changed color, and it served as the exogenous cue. The onset circle was completely irrelevant to the task, never contained the response target, and was not predictive of the response target's location. Since the onset never contained the response target, any saccades made to the onset are presumed to be involun- 


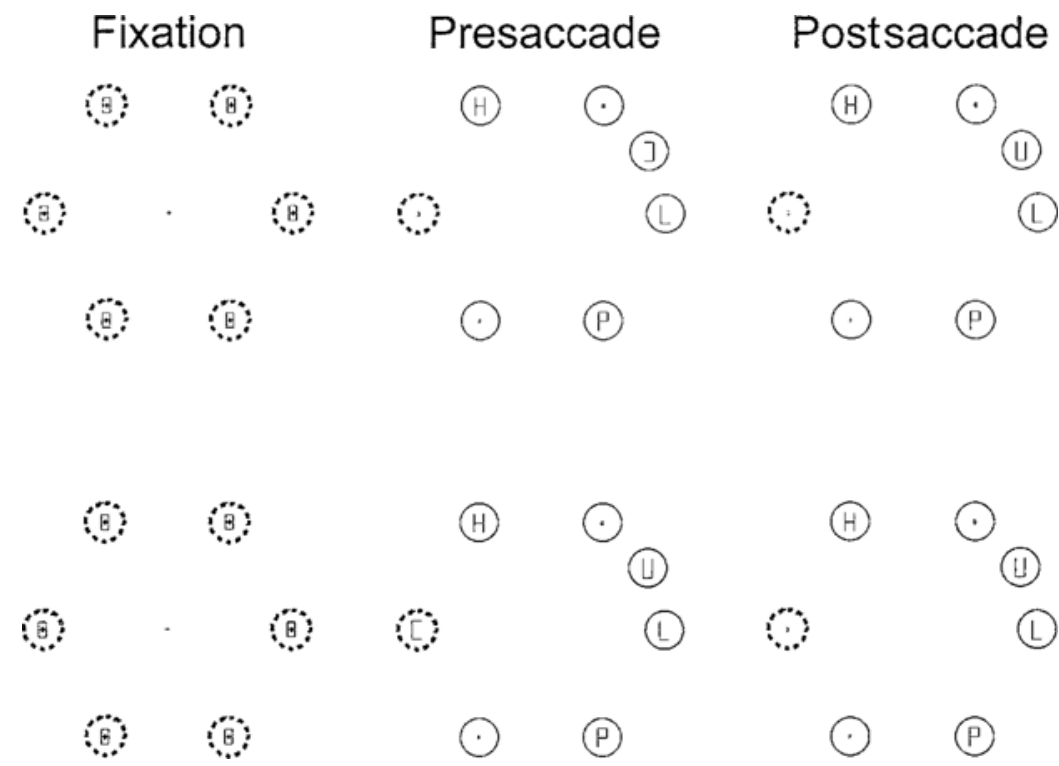

Figure 1. Examples of the displays used in Experiment 1. The dashed lines represent white, and the solid lines represent red. In the presaccade display, all of the distractors changed from white to red and the target was the remaining white color singleton ( 9 o'clock position in both examples). The onset is at the 2 o'clock position. The top sequence shows a response-compatible probe at the location of the onset. The bottom sequence shows a response-incompatible probe at the location of the target.

tary in nature. To measure covert attention, we presented response compatible or incompatible probes at the location of the onset or the saccade target before the eyes began to move. If the identity (compatibility) of the probe affected RTs to the response target, then we can assume that attention had been allocated to its location. That is, the probe allowed us to measure where covert attention was focused after the signal (color change) to move the eyes had been given but before the eyes had begun to move. Unlike the response target itself, the probe was made large enough so that it could be identified while fixating the center of the screen and, therefore, was identifiable before the eyes began to move. Critical to these experiments is the fact that the probes were visible only between the time of the presentation of the cue and the initiation of the eye movement - all probes were replaced by neutral items or the target (in the case of probes presented at the target location) before or during the initial saccade. This allowed us to track the location, and in Experiment 2 , the time course of attention before the eyes began to move.

To measure overt attention, we examined the landing point of the initial and secondary saccades. By examining both saccades, we can distinguish between several models of overt and, taken with the response compatibility data, covert orienting. For example, one possibility is that the exogenous signal from the onset always captures attention but that sometimes the endogenous signal generated in response to the color singleton is generated more quickly. If this is the case, we might ex- pect that the initial saccade lands on the onset, followed by a corrective saccade to the target. The absence of a secondary saccade to the onset would suggest that on that trial the onset was unable to capture overt attention. Likewise, if capture occurs because on some trials the endogenous signal develops more slowly than does the exogenous signal, we might expect the eyes to go to the onset followed by a quick corrective saccade to the color singleton saccade target (Theeuwes et al., 1998). Of course, if eye movements and covert attention are coupled, we should find the pattern of initial and secondary saccades reflected in the response compatibility data.

Note that for Experiments 1 and 2, we will refer to the color singleton circle as the saccade target, whereas the letter that is responded to will be referred to as the response target. Although the saccade target always contained the response target when the eyes landed on it, in some conditions the saccade target could contain a neutral item or probe before the eyes moved.

\section{EXPERIMENT 1}

\section{Method}

Participants. Sixteen students from the University of Illinois were paid to participate in Experiment 1 ( 8 males, 8 females; average age, 22.4 years).

Stimuli. A Pentium 133-MHz computer with a 19-in. color monitor running at $60 \mathrm{~Hz}$ was used to present the stimuli and record participants' manual RTs. Eye movements were recorded with an Eyelink tracker (SR Research Ltd.) with $250-\mathrm{Hz}$ temporal resolution and $0.2^{\circ}$ spatial resolution. A chinrest was used to stabilize head 
position, and the participants viewed the screen from a distance of $53.3 \mathrm{~cm}$

The stimuli initially consisted of six white circles on a black background arranged around the perimeter of an imaginary circle $25.4^{\circ}$ in diameter (see Figure 1). Each circle was $3.81^{\circ}$ in diameter and contained double figure- 8 premasks. The premasks were $0.25^{\circ}$ wide $\times 0.51^{\circ}$ tall and $1.21^{\circ}$ wide $\times 2.41^{\circ}$ tall, and their sizes mirrored the two possible sizes of stimuli. The larger stimuli were large enough to be identified while the eyes were fixating the central fixation cross (verified in pilot testing). The smaller stimuli, similar to those used by Theeuwes et al. (1998), were too small to be identified from central fixation and necessitated an eye movement to their location in order to be identified. The neutral letters consisted of F, P, S, H, U, L, and O.

The target and probes were always a $\mathrm{C}$ or a mirror-reversed $\mathrm{C}$. The participants responded to the target by pressing one of two keys on the computer keyboard. In the response-compatible condition, the probe matched the identity of the response target, whereas in the response-incompatible condition, the probe was mapped to the response opposite to that for the target.

Procedure. The time course of events is shown in Figure 1. Each trial began with the presentation of a fixation cross. The participants fixated the cross and pressed the space bar to continue with the trial. The software allowed a trial to continue only if the participant was fixating within $2.5^{\circ}$ of the cross when the space bar was pressed. The premasks and circles (see the fixation screen in Figure 1) appeared $700 \mathrm{msec}$ after the space bar was pressed and were colored white. After a delay of $1,000 \mathrm{msec}$, the fixation cross was removed, all but one of the white circles changed to red, and line segments were removed from the premasks to reveal the stimulus letters (see the presaccade screen in Figure 1). The remaining white circle, a color singleton, was the saccade target. In addition, the onset appeared as a new letter and red circle at the $2,4,8$, or 10 o'clock position and was separated from the saccade target by 90 or 150 radial degrees. If the participant moved his or her eyes before the fixation cross was removed, a buzzer sounded and the trial was aborted.

The stimulus letters consisted of six of the seven possible neutral letters and one probe letter. The probe letter occurred in the onset or target circle, was always large, and consisted of a $\mathrm{C}$ or a mirrorreversed $\mathrm{C}$ that was either response compatible or incompatible with the response target. To prevent spatial frequency differences between the small probe and the large target from potentially attracting attention, the neutral letters were randomly assigned to the large or small size. In Experiment 1, the probe was removed and replaced with either a neutral item (when it occurred in the onset) or the response target (when it occurred in the white circle) during the initial saccade (see the postsaccade screen in Figure 1). A saccade was detected on line if the eyes moved at least $2.5^{\circ}$ from the fixation point and exceeded a velocity of $110 \mathrm{deg} / \mathrm{sec}$ ( 7 pixels per $4-$ msec sample). The saccade detection algorithm was designed to minimize the latency between saccade detection and stimulus change on the screen. More accurate saccade analyses were performed off line using the Eyelink software. In the off-line analysis, an eye movement was classified as a saccade when its distance exceeded $0.2^{\circ}$ and its velocity reached $30 \mathrm{deg} / \mathrm{sec}$, or when its distance exceeded $0.2^{\circ}$ and its acceleration reached $9,500 \mathrm{deg} / \mathrm{sec}^{2}$. Only trials in which the probe change occurred after the saccade had been initiated and before the saccade was terminated were accepted for analysis. How quickly the probe could be changed is a function of the time to detect a saccade, which is partially dependent on the latency between the host and stimulus computers $(10 \mathrm{msec})$ and the current position of the scan line in the CRT. For the accepted trials across Experiments 1 and 2, saccade durations averaged $45 \mathrm{msec}$ and saccades were detected $23 \mathrm{msec}$ after the saccade started. Given the $60-\mathrm{Hz}$ refresh rate of the display, probes were changed, on average, between 23 and $38 \mathrm{msec}$ after the saccade began (22 to $7 \mathrm{msec}$ before the saccade terminated).
The participants were instructed to move their eyes to the sole white circle and to determine whether a $\mathrm{C}$ or a mirror-reversed $\mathrm{C}$ was contained within it. The participants responded by pressing the " $Z$ " key if the saccade target contained a mirror-reversed $\mathrm{C}$ and the "/" key for a forward C. A tone sounded if an incorrect key was pressed or if the eyes moved from fixation before the color singleton appeared.

Experiment 1 was approximately $1 \mathrm{~h}$ long and consisted of 640 trials.

\section{Results}

For both experiments, only RTs from correctly answered trials were analyzed ( $98 \%$ of trials). Because the on-line saccade detection algorithm was optimized for speed, some saccades were not detected and the probe change occurred after the eyes had stopped moving. Only trials in which the probe change occurred during the initial saccade were included in the analyses ( $9 \%$ rejected). Outliers were removed by excluding all RTs greater than twice the mean of each cell (1\%). Response compatibility effects for each condition were calculated by subtracting the mean RT for compatible trials from the mean RT for incompatible trials for each participant.

Initial and secondary saccades were analyzed using paired $t$ tests. Saccades counted as landing on either item of interest if they landed within a 30 radial degree pie slice centered on the item of interest and landed outside the $2.5^{\circ}$ exclusion zone around central fixation. One participant's data were excluded from analysis because no secondary saccades were made from the saccade target to the onset. The initial saccade was more likely to go to the saccade target than to the onset $[M=.59$ and .30 , respectively; $t(14)=2.6, p<.05]$, indicating that the onsets did not always capture the eyes. ${ }^{2}$ In addition, saccadic latencies were shorter when the eyes went to the onset than when they went to the saccade target $[M=$ 204 and $233 \mathrm{msec}$, respectively; $t(14)=13.0, p<.01$ ], suggesting that saccadic programming finished earlier when the eyes went to the onset.

Secondary saccades occurred on trials in which the initial saccade went to the saccade target or the onset and then went to the other item of interest (onset or saccade target, respectively). Secondary saccades were much more likely to occur from the onset to the saccade target than from the saccade target to the onset $[M=.88$ and .04 , respectively; $t(14)=25.8, p<.01]$. In contrast to the initial saccades, secondary saccades to the onset (from the saccade target) were much slower than those to the saccade target (from the onset) $[M=377$ and $96 \mathrm{msec}$, respectively; $t(14)=8.2, p<.01]$. The relative abundance of secondary saccades to the saccade target and their much quicker nature suggests that their programming might have already occurred before the eyes landed on the onset (McPeek, Skavenski, \& Nakayama, 2000; Theeuwes et al., 1998).

RTs were sorted on the basis of whether the initial saccade went to the target color singleton or to the taskirrelevant onset. Because the trials in which the eyes went initially to the onset included an extra corrective saccade to the saccade target, for all experiments sepa- 
rate response compatibility analyses were performed for each initial saccade direction.

Figure 2 shows the response compatibility effects for each probe location when the eyes initially went to the saccade target or to the onset, ignoring the direction of the secondary saccade. Table 1 shows the raw means. When the eyes went to the saccade target, only probes that occurred at the saccade target position produced a significant response compatibility effect $[t(15)=4.44, p<$ $.01 ; M=39.6 \mathrm{msec}$, suggesting that probes that occurred at the onset were not attended when the eyes went to the saccade target. When the eyes went to the onset, probes that occurred both at the saccade target $[t(15)=$ $3.90, p<.01 ; M=50.5 \mathrm{msec}]$ and at the onset $[t(15)=$ 2.54, $p<.01 ; M=-25.1 \mathrm{msec}]$ produced significant compatibility effects, suggesting that both items were attended before the eyes had started to move. Interestingly, when the eyes went to the onsets, probes at the onset produced a negative compatibility effect. This will be discussed in greater detail in the Discussion section.

\section{Discussion}

Experiment 1 demonstrated that when the eyes go to the saccade target, only probes at the location of the saccade target affect responses. However, when the eyes go to the onset, items at the onset location and at the intended target location affect RTs, indicating that covert attention can go to either location before the involuntary eye movement begins. These results might suggest that covert attention and voluntary eye movements are tightly coupled, whereas covert attention and involuntary eye movements are not.

An alternative explanation is that these results reflect the differing time courses of the endogenous and exoge- nous attentional systems and that the two systems compete, or race, to win control of the eyes (Findlay \& Walker, 1999; Kramer et al., 2001). In this horse race model, when the eyes go to the onset, the exogenous system has won control of the eyes, possibly because it generated a signal to shift attention more quickly than did the endogenous system (Cheal \& Lyon, 1991; Juola, Koshino, \& Warner, 1995; Müller \& Rabbitt, 1989; Trappenberg, Dorris, Munoz, \& Klein, 2001). However, there might be a considerable delay between winning the competition for control of the eyes and the execution of the eye movement. If the delay for executing a covert attention shift is shorter than is the delay for an overt shift (Cheal \& Lyon, 1991; Juola et al., 1995; Müller \& Rabbitt, 1989; Trappenberg et al., 2001), then the losing endogenous signal might be able to shift covert attention while the overt shift triggered by the exogenous signal is still being programmed. Support for this is found in the latencies of the secondary saccades, in which the eyes initially went to the onset and then made a secondary corrective saccade toward the saccade target. These latencies are considerably shorter than those of the primary saccades, which suggests that some programming occurred either during the previous eye movement or even before the previous saccade had been triggered.

Within this framework, the lack of any effect for probes at the onset location when the eyes go to the saccade target suggests that, in these instances, an exogenous signal has failed to take control. That is, in terms of our horse race analogy, if an exogenous signal fails to win the race for control of the eyes, it might still have a chance to take control of covert attention and shift attention toward the onset before the eyes have moved. However, in Experiment 1 , there was no evidence of any processing of onset

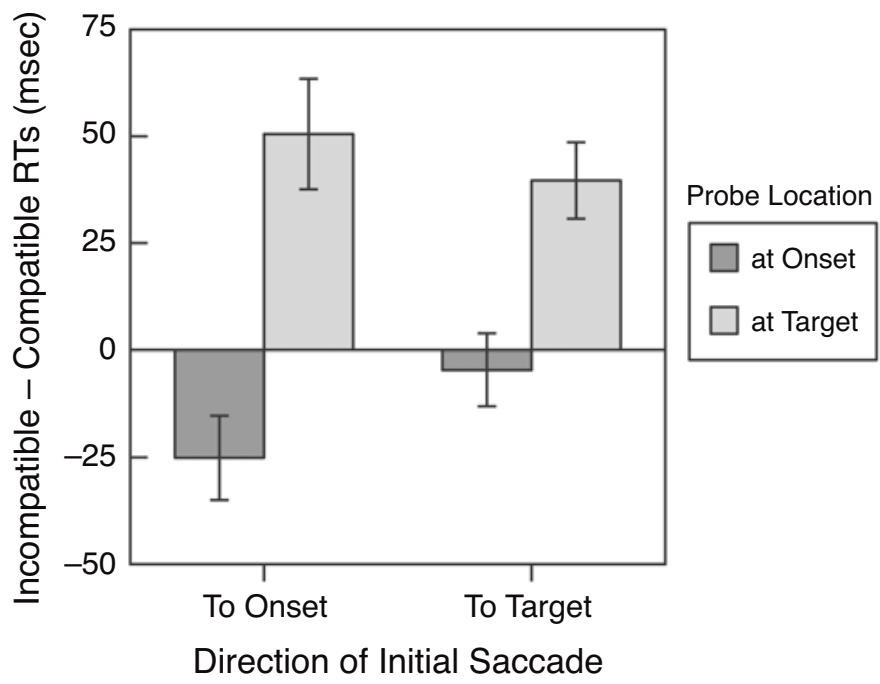

Figure 2. Response compatibility effects for Experiment 1. Response compatibility effects were calculated by subtracting the mean response times (RTs) for trials in which the probe was response compatible with the target from the mean RTs for those in which the probe was response incompatible with the target [RT(I) - RT(C)]. 
Table 1

Mean Response Times (in Milliseconds) for Experiments 1 and 2

\begin{tabular}{|c|c|c|c|c|c|}
\hline $\begin{array}{l}\text { Probe } \\
\text { Time }\end{array}$ & $\begin{array}{c}\text { Saccade } \\
\text { Direction }\end{array}$ & $\begin{array}{c}\text { Probe } \\
\text { Location }\end{array}$ & Incompatible & Compatible & Difference \\
\hline \multicolumn{6}{|c|}{ Experiment 1} \\
\hline & To onset & At onset & 905.4 & 930.5 & -25.1 \\
\hline & To onset & At target & 895.5 & 845.0 & 50.5 \\
\hline & To target & At onset & 809.0 & 813.6 & -4.6 \\
\hline & To target & At target & 800.9 & 761.2 & 39.6 \\
\hline \multicolumn{6}{|c|}{ Experiment 2} \\
\hline Early & To onset & At onset & 904.0 & 926.0 & -22.0 \\
\hline Early & To onset & At target & 919.1 & 920.0 & -1.0 \\
\hline Early & To target & At onset & 840.3 & 840.1 & 0.1 \\
\hline Early & To target & At target & 822.7 & 838.6 & -15.9 \\
\hline Late & To onset & At onset & 906.0 & 923.4 & -17.3 \\
\hline Late & To onset & At target & 898.1 & 861.8 & 36.2 \\
\hline Late & To target & At onset & 836.3 & 833.7 & 2.6 \\
\hline Late & To target & At target & 821.9 & 777.6 & 44.3 \\
\hline \multicolumn{6}{|c|}{ Percentage of Saccades } \\
\hline & Direction & \multicolumn{2}{|c|}{ Exp. 1} & xp. 2 & \\
\hline & To onset & \multicolumn{2}{|c|}{30} & 21 & \\
\hline & To target & \multicolumn{2}{|c|}{59} & 0 & \\
\hline
\end{tabular}

probes when the eyes went to the saccade target. This suggests that, when faced with onsets, the exogenous system either generates a fast signal that wins the race for control or generates no signal at all. Some evidence for this fast exogenous signal can be seen in the saccade latencies, in which saccades to onsets occur on average 29 msec sooner than do saccades to targets.

The goal of Experiment 2 was to determine whether the compatibility effects observed when the eyes went to the onset and the probe was at the saccade target location are caused by covert attention shifting from the onset to the saccade target before the eyes move, as is predicted by our horse race model. When covert attention is drawn to an onset, it typically peaks within $100 \mathrm{msec}$, whereas voluntary shifts following the presentation of a symbolic cue typically peak within 200 to 300 msec (Cheal \& Lyon, 1991; Müller \& Rabbitt, 1989). To determine whether covert attention shifts from the onset to the saccade target prior to the saccade are possible, probes in Experiment 2 were visible only during the presentation of the onset and the saccade target or following the first $100 \mathrm{msec}$ afterward. Probing during two different time periods should allow us to track covert attention if it systematically shifts around the display. If the compatibility effects observed when the eyes went to the onset were simply caused by a decoupling from the outset of involuntary eye movements and the targeting of covert attention shifts, then we should find that compatibility effects are the same across the time course. In contrast, if covert attention shifts from the onset to the saccade target when the eyes go to the onset, we should find that the compatibility effects at the different locations change across time. For example, a compatibility effect might be found at the onset location early during processing but might shift to the location of the saccade target late in processing.

\section{EXPERIMENT 2}

\section{Method}

Participants. Thirty students from the University of Illinois were paid to participate in Experiment 2 (30 participants). In Experiment 2 , four participants were excluded from data analysis because they failed to make any saccades to the onset in some cells. Of the 26 remaining participants ( 11 males, 15 females), the average age of the participants was 22.3 years.

Procedure. The procedure was identical to that used in Experiment 1 except that two probe presentation times were used. The early probes were visible for the first $100 \mathrm{msec}$ after the color change - after the first $100 \mathrm{msec}$, the probe changed into a neutral item (when it occurred in the onset) or the response target (when it occurred at the color singleton target location). The late probe was presented by changing one of the neutral items into the probe $100 \mathrm{msec}$ after the color change. In the case of the late probe, the saccade target circle contained a neutral item for the first $100 \mathrm{msec}$. The probe then changed into a neutral item or the response target when the eyes began to move, depending on whether the probe occurred at the onset or color singleton target location, respectively. As in Experiment 1, the response target was smaller than the probe was, and it was too small to be identified with peripheral vision.

Experiment 2 was approximately $1 \mathrm{~h}$ long and consisted of 640 trials.

\section{Results}

As in Experiment 1, initial saccades to the onset were less frequent than those to the saccade target $[M=.21$ and .60 , respectively; $t(25)=6, p<.01]$ and had a shorter latency $[M=217$ and $262 \mathrm{msec}$, respectively; $t(25)=7.3$, $p<.01]$. The remaining $19 \%$ of the saccades landed on an item other than the onset or saccade target. Likewise, the secondary saccades mirrored the results of Experiment 1, with secondary saccades more likely to go to the saccade target (from the onset) than to the onset (from the saccade target) $[M=.82$ and .03 , respectively; $t(25)=21.9, p<$ .01]. Likewise, secondary saccades to the saccade target had a much shorter latency than did those to the onset, again suggesting that the secondary saccade to the saccade target had been programmed even before the eyes had landed on the onset $[M=106$ and $393 \mathrm{msec}$, respectively; $t(25)=10.5, p<.01]$.

Figure 3 shows the response compatibility effects for each probe location and sampling period when the eyes went to the saccade target or to the onset. Late probes yielded a pattern of responses identical to that in Experiment 1: When the eyes went to the saccade target, only probes at the saccade target $[t(25)=9.9, p<.01]$ showed a compatibility effect, whereas when the eyes went to the onsets, probes at the saccade target $[t(25)=4.9, p<$ $.01]$ and at the onset $[t(25)=3.2, p<.01]$ both affected RTs. However, when the probe occurred during the first $100 \mathrm{msec}$, only probes that occurred at the eventual landing spot of the first saccade affected RTs $[t(25)=4.1$, $p<.01$ for target probes when the eyes went to the saccade target, and $t(25)=3.2, p<.01$ for onset probes when the eyes went to the onset]. This suggests that covert attention initially heads in the direction of the saccade but may later change direction after the first $100 \mathrm{msec}$. 


\section{Discussion}

Although several studies have suggested that covert attention and voluntary eye movements are tightly coupled (Deubel \& Schneider, 1996; Hoffman \& Subramaniam, 1995; Irwin \& Gordon, 1998; Kowler et al., 1995), there has been some debate as to whether eye movements and covert attention are linked when gaze shifts involuntarily. The results of Experiments 1 and 2 suggest that covert attention can move to a location other than the goal of the immediate saccade, but only when the eyes are captured by an exogenous onset stimulus during the programming of a saccade to an endogenous stimulus; under these circumstances, covert attention moves first to the location of the exogenous stimulus (the onset) before moving to the location of the endogenous stimulus (the target) before any saccade is generated. However, this independent shift of covert attention is possible only after at least $100 \mathrm{msec}$ have elapsed: within the first $100 \mathrm{msec}$ following a signal to shift attention, covert attention and eye movements are coupled, with covert attention going only to the goal of the immediate saccade. These findings not only support the oculomotor readiness (Klein, 1980) and premotor (Rizzolatti \& Craighero, 1998; Rizzolatti, Riggio, \& Sheliga, 1994; Umiltà, Riggio, Dascola, \& Rizzolatti, 1991) hypotheses, which state that the programming of a saccade initiates a shift of covert attention, but also indicate that eye movements are a valid measure for determining the target of covert attention.

Our results also suggest that onsets, at least with the types of displays used here, capture covert attention on only a small proportion of trials (Martin-Emerson \& Kramer, 1997; Miller, 1989). Averaged across experiments, the data show that the eyes went to the onset on only $26 \%$ of the trials. Given that eye movements and the initial movement of covert attention appear to be linked (onset probes never affected responses when the eyes went to the saccade target), these results, along with the results of Peterson et al. (2002) that showed marginal oculomotor capture by irrelevant onsets when the target was a nonsingleton and no endogenous cue was present, would suggest that onsets capture attention only weakly.

\section{A MODEL OF COVERT ATTENTION AND SACCADE GENERATION}

At first glance, the results of these experiments suggest that in some instances, such as when the eyes make an involuntary movement, covert and overt attention can become decoupled. However, on closer inspection, the route that covert attention takes mimics the path that the eyes will take. For example, in trials in which the eyes are captured by an onset, covert attention first shifts to the onset and then to the intended target. This is followed a short time later by a series of eye movements first to the onset and then to the saccade target. Likewise, when the eyes go directly to the intended target, evidence of covert shifts are found only at the location of the saccade target.

One way to explain these data is to imagine a simple horse race model, in which two signals, exogenous and endogenous, race to control the orienting of attention. The first process to finish controls where attention was directed first. The second-place finisher then waits for the winner's orders to be carried out before it is free to take control (assuming that the second signal is not extinguished). If covert shifts take less time to set up than overt shifts do, as would be suggested by the significant

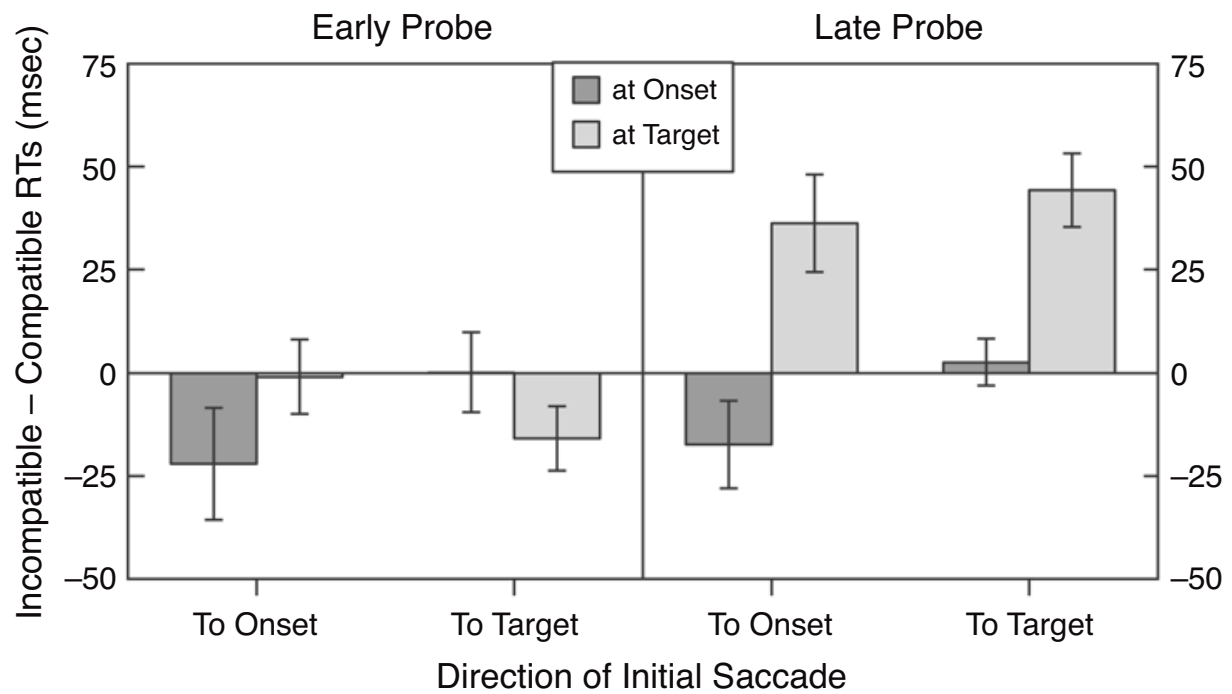

Figure 3. Response compatibility effects for Experiment 2. Response compatibility effects were calculated by subtracting the mean response times (RTs) for trials in which the probe was response compatible with the target from the mean RTs for those in which the probe was response incompatible with the target $[\mathrm{RT}(\mathrm{I})-\mathrm{RT}(\mathrm{C})]$. 
compatibility effects before the eyes even begin to move, then this simple race model predicts that covert attention switches from the saccade target to the target of the second-place finisher. For example, when the eyes go to the target, covert attention should first shift to the target and then shift to the onset.

However, this simple horse race model is unable to predict the results of Experiments 1 and 2. More specifically, when the eyes went to the target, there was no evidence that covert attention had visited the onset. One possibility is that when the endogenous signal wins the race for control, it blocks all exogenous signals for some period of time (Bacon \& Egeth, 1994; Juola et al., 1995; Theeuwes, Kramer, Hahn, Irwin, \& Zelinsky, 1999). Support for this is seen in the relative scarcity of secondary saccades to the onset and their relatively long latencies. However, if we assume that the saccade latencies represent the finishing times in the race, then this type of model predicts that onsets should capture attention on a majority of trials. For example, on the basis of the distribution of saccade latencies for Experiment 1, onsets should capture attention $88 \%$ of the time, whereas the observed degree of capture is only $30 \%$. A more likely possibility is that the eyes initially go to the target only in instances in which no exogenous signal has been generated. That is, the exogenous system either generates a fast signal that is always capable of winning the race for control or it generates no signal at all. Either possibility suggests that onsets do not always automatically capture attention. ${ }^{3}$

One additional aspect of the data requires comment. Whenever the eyes went to the onset (see Figures 2 and 3 ), a reverse compatibility effect was obtained. That is, responses were faster when the probe was incompatible with the response to the target than when the probe was compatible. This effect was also observed when the eyes went to the target and the probe was presented within the first $100 \mathrm{msec}$ (see the left panel of Figure 3). Recent studies by Eimer and Schlaghecken (2002; see also Eimer \& Schlaghecken, 1998a, 1998b) provide a possible explanation for this seemingly paradoxical finding. In their studies, participants made speeded responses to a central target that was preceded by a masked prime (analogous to our probe). Eimer and Schlaghecken found that the direction of the response compatibility effect is a function of stimulus visibility or stimulus quality (manipulated by varying how long the prime was visible before the presentation of the mask). Specifically, as the stimulus presentation duration increased, the response compatibility effect crossed over from negative to positive. Interestingly, the crossover point for the compatibility effect was nearly identical to the point at which participants were able to consciously recall the target. One possible explanation is that responses to subliminal stimuli are automatically inhibited (Eimer \& Schlaghecken, 2002; but see also Marcel, 1983, and Cheesman \& Merikle, 1984, for evidence that subliminal stimuli that prime semantic or perceptual representations lead to positive priming). If the inverse compatibility effect is attributed to poor stimulus quality, then our hypothesis - that when the eyes go to the onset, covert attention briefly visits the onset before switching to the targetwould be supported. In our case, the brief visit of covert attention leads to poor stimulus processing and an inverse compatibility effect. The results of Experiment 2 support this notion: When the eyes go to the target, the early target probe presentation produces an inverse compatibility effect whereas the later presentation produces a positive effect. The mean saccade latency in Experiment 2 was $262 \mathrm{msec}$, which means that, on average, in the late probe condition the probe would have been "visible" to attention for $162 \mathrm{msec}$, whereas in the early probe condition it would have been visible for only $100 \mathrm{msec}$. Given that covert attentional shifts are not instantaneous (Cheal \& Lyon, 1991), the early probe would have been visible to attention for less than $100 \mathrm{msec}$. Given these attentional dwell times, this suggests that the inverse compatibility effect in the early probe condition was caused by insufficient attentional processing.

In conclusion, the results of the experiments presented here suggest that covert attention always precedes a saccade to the saccade target. This means that the landing point of the saccade is a valid indicator of the initial aim of covert attention, and therefore eye movements are a more powerful measure of covert attention than are manual RTs or error rates. Given that, our finding that saccades were made to the onset on only a minority of trials suggests that the sudden appearance of an onset does not lead to an obligatory capture of attention, whether covert or overt.

\section{REFERENCES}

Bacon, W. F., \& Egeth, H. E. (1994). Overriding stimulus-driven attentional capture. Perception \& Psychophysics, 55, 485-496.

Cheal, M. L., \& LyON, D. R. (1991). Central and peripheral precuing of forced-choice discrimination. Quarterly Journal of Experimental Psychology, 43A, 859-880.

Cheesman, J., \& Merikle, P. M. (1984). Priming with and without awareness. Perception \& Psychophysics, 36, 387-395.

Deubel, H., \& SchNeIDER, W. X. (1996). Saccade target selection and object recognition: Evidence for a common attentional mechanism. Visual Research, 36, 1827-1837.

EIMER, M., \& SCHLAGHECKEN, F. (1998a). Effects of masked stimuli on motor activation: Behavioral and electrophysiological evidence. Journal of Experimental Psychology: Human Perception \& Performance, 24, 1737-1747.

EiMER, M., \& SCHLAGHECKEN, F. (1998b). Response facilitation and inhibition in manual, vocal, and oculomotor performance: Evidence for a modality-unspecific mechanism. Journal of Motor Behavior, $\mathbf{3 3}_{2}$ $16-26$.

Eimer, M., \& Schlaghecken, F. (2002). Links between conscious awareness and response inhibition: Evidence from masked priming. Psychonomic Bulletin \& Review, 9, 514-520.

FindLAY, J. M., \& WaLKer, R. (1999). A model of saccade generation based on parallel processing and competitive inhibition. Behavioral \& Brain Sciences, 22, 661-721.

GoDIJ, R., \& THEEUWES, J. (2002). Programming of endogenous and exogenous saccades: Evidence for a competitive integration model. Journal of Experimental Psychology: Human Perception \& Performance, 28, 1039-1054. 
Henderson, J. M. (1992). Visual attention and eye movement control during reading and scene perception. In K. Rayner (Ed.), Eye movements and visual cognition: Scene perception and reading (pp. 260283). New York: Springer-Verlag.

Henderson, J. M., \& Hollingworth, A. (1999). The role of fixation position in detecting scene changes across saccades. Psychological Science, 10, 438-443.

Henderson, J. M., Pollatsek, A., \& Rayner, K. (1989). Covert visual attention and extrafoveal information use during object identification. Perception \& Psychophysics, 45, 196-208.

Hoffman, J. E., \& Subramaniam, B. (1995). The role of visual attention in saccadic eye movements. Perception \& Psychophysics, $\mathbf{5 7}$, 787-795.

Irwin, D. E., Colcombe, A. M., Kramer, A. F., \& Hahn, S. (2000). Attentional and oculomotor capture by onset, luminance, and color singletons. Vision Research, 40, 1443-1458.

IRWIN, D. E., \& GORDON, R. D. (1998). Eye movements, attention, and transsaccadic memory. Visual Cognition, 5, 127-155.

Juola, J. F., Koshino, H., \& Warner, C. B. (1995). Tradeoffs between attentional effects of spatial cues and abrupt onsets. Perception \& Psychophysics, 57, 333-342.

KLEIN, R. (1980). Does oculomotor readiness mediate cognitive control of visual attention? In R. Nickerson (Ed.), Attention and performance III (pp. 259-276). Hillsdale, NJ: Erlbaum.

Kowler, E., Anderson, E., Dosher, B., \& Blaser, E. (1995). The role of attention in the programming of saccades. Vision Research, $\mathbf{3 5}_{2}$ 1897-1916.

Kramer, A. F., Cassavaugh, N. D., Irwin, D. E., Peterson, M. S., \& HAHN, S. (2001). Influence of single and multiple onset distractors on visual search for singleton targets. Perception \& Psychophysics, $\mathbf{6 3}$, 952-968.

MARCEL, A. J. (1983). Conscious and unconscious perception: Experiments on visual masking and word recognition. Cognitive Psychology, 15, 197-237.

Martin-Emerson, R., \& Kramer, A. F. (1997). Offset transients modulate attentional capture by sudden onsets. Perception \& Psychophysics, 59, 739-751.

McPeek, R. M., Skavenski, A. A., \& Nakayama, K. (2000). Concurrent processing of saccades. Vision Research, 40, 2499-2516.

Miller, J. (1989). The control of attention by abrupt visual onsets and offsets. Perception \& Psychophysics, 45, 567-571.

MoKLer, A., \& Fischer, B. (1999). The recognition and correction of involuntary prosaccades in an antisaccade task. Experimental Brain Research, 125, 511-516.

Müller, H. J., \& RabbitT, P. M. A. (1989). Reflexive and voluntary orienting of visual attention: Time course of activation and resistance to interruption. Journal of Experimental Psychology: Human Perception \& Performance, 15, 315-330.

Peterson, M. S., Kramer, A. F., Irwin, D. E., \& Hahn, S. (2002). Modulation of oculomotor capture by abrupt onsets during attentionally demanding visual search. Visual Cognition, 9, 755-791.

Rayner, K., McConkie, G. W., \& Ehrlich, S. (1978). Eye movements and integrating information across fixations. Journal of Experimental Psychology: Human Perception \& Performance, 4, 529-544.

Rizzolatti, G., \& Craighero, L. (1998). Spatial attention: Mechanisms and theories. In M. Sabourin, F. I. M. Craik, \& M. Robert (Eds.), Advances in psychological science: Vol. 2. Biological and cognitive aspects (pp. 171-198). Hove, U.K.: Psychology Press.

Rizzolatti, G., Riggio, L., \& Sheliga, B. M. (1994). Space and selective attention. In C. Umiltà \& M. Moscovitch (Eds.), Attention and performance $X V$ : Conscious and nonconscious information processing (pp. 232-265). Cambridge, MA: MIT Press, Bradford Books.

TheeUwes, J. (1991). Exogenous and endogenous control of attention: The effect of visual onsets and offsets. Perception \& Psychophysics, 49, 83-90.
Theeuwes, J., Kramer, A. F., Hahn, S., \& Irwin, D. E. (1998). Our eyes do not always go where we want them to go: Capture of the eyes by new objects. Psychological Science, 9, 379-385.

Theeuwes, J., Kramer, A. F., Hahn, S., Irwin, D. E., \& Zelinsky, G. J. (1999). Influence of attentional capture on oculomotor control. Journal of Experimental Psychology: Human Perception \& Performance, 25, 1595-1608.

Trappenberg, T. P., Dorris, M. C., Munoz, D. P., \& Klein, R. M. (2001). A model of saccade initiation based on the competitive integration of exogenous and endogenous signals in the superior colliculus. Journal of Cognitive Neuroscience, 13, 256-271.

Umiltà, C., Riggio, L., Dascola, I., \& Rizzolatti, G. (1991). Differential effects of central and peripheral cues on the reorienting of spatial attention. European Journal of Cognitive Psychology, 3, 247-267.

Warner, C. B., Juola, J. F., \& Koshino, H. (1990). Voluntary allocation versus automatic capture of visual attention. Perception \& Psychophysics, 48, 243-251.

YANTIS, S., \& JONIDES, J. (1984). Abrupt visual onsets and selective attention: Evidence from visual search. Journal of Experimental Psychology: Human Perception \& Performance, 10, 601-621.

\section{NOTES}

1. Note that attentional capture by onsets appears to occur only when attention is unfocused. For example, when subjects are allowed to focus their attention in advance of the appearance of an onset, capture does not occur (Theeuwes, 1991). In the extreme case, it is possible after several days of training to learn to use the onset as a cue to orient in the opposite direction (Warner, Juola, \& Koshino, 1990).

2. One possibility is that because the probe shared the identity with the target, some of the saccades made to the onset might have been driven by the probe rather than by the transient nature of the onset. In that case, more saccades should have been made to the onset when it contained a probe than when it contained a neutral letter. Likewise, if the probe influenced eye movements, more saccades should have been made to the target when it initially contained the large probe than when it contained the small target letter. A two-way analysis of variance with initial saccade target (onset or target) and probe location (in the saccade target or elsewhere) showed no main effect of probe location $[F(1,15)<$ $1]$, suggesting that the probe had no effect on saccade direction and that saccades to the onset or target were driven purely by the onset or target, respectively. Likewise, probe location and saccade direction failed to interact $[F(1,15)=1.3, p>.10]$. Similar results were found for the early probe in Experiment $2[F(1,25)=1.5$ and $1.7, p>.10$ for the main effect and interaction, respectively].

In addition, in both experiments, the proportion of saccades made to the onset was greater than that expected by chance alone. For example, in Experiment 1, 59\% of the saccades landed on the target and $30 \%$ landed on the onset. If saccades that did not land on the target were randomly guided to one of the other stimulus locations, then the expected probability of landing on the onset would have been $6.83 \%$ (one sixth of the remaining $41 \%$ ).

3. The competitive integration model of saccade generation (Godijn $\&$ Theeuwes, 2002) would predict similar results. However, to test the competitive integration model, we would need to include a condition in which the target and onset were separated by 30 radial degrees. Because we were unsure of the spatial precision of covert attention and wanted to maximize the effect size of the response compatibility data, we elected to use $90^{\circ}$ and $150^{\circ}$ separations

(Manuscript received January 10, 2003; revision accepted for publication May 23, 2003.) 